\title{
Oscillatory Behavior of Solutions of Certain Integrodynamic Equations of Second Order on Time Scales
}

\author{
Said R. Grace ${ }^{1}$ and Mohamed A. El-Beltagy ${ }^{1,2}$ \\ ${ }^{1}$ Engineering Mathematics Department, Engineering Faculty, Cairo University, Giza 1221, Egypt \\ ${ }^{2}$ Electrical and Computer Engineering Department, Engineering Faculty, Effat University, Jeddah 21478, Saudi Arabia \\ Correspondence should be addressed to Mohamed A. El-Beltagy; zbeltagy@hotmail.com
}

Received 27 April 2014; Revised 29 June 2014; Accepted 29 June 2014; Published 9 July 2014

Academic Editor: Tongxing Li

Copyright (C) 2014 S. R. Grace and M. A. El-Beltagy. This is an open access article distributed under the Creative Commons Attribution License, which permits unrestricted use, distribution, and reproduction in any medium, provided the original work is properly cited.

\begin{abstract}
This paper deals with the oscillatory behavior of forced second-order integrodynamic equations on time scales. The results are new for the continuous and discrete cases and can be applied to Volterra integral equation on time scale. We also provide a numerical example in the continuous case to illustrate the results.
\end{abstract}

\section{Introduction}

Dynamic equations on time scales is a fairly new topic and for general basic ideas and background, we refer the reader to [1]. Oscillation and nonoscillation results for integral equations of Volterra type have been discussed only in a few papers in the literature; see [2-5].

In this paper, we are concerned with the oscillatory behavior of solutions of the second-order integrodynamic equation on time scales of the form

$$
\left(r(t) x^{\Delta}(t)\right)^{\Delta}+\int_{0}^{t} a(t, s) F(s, x(s)) \Delta s=e(t) .
$$

We take $T \subseteq R$ to be an arbitrary time scale with $0 \in T$ and $\operatorname{Sup} T=\infty$. Whenever we write $t \geq s$, we mean $t \in[s, \infty) \cap T$. We assume throughout the following.

(I) $e, r: T \rightarrow R$ and $a: T \times T \rightarrow R$ are rd-continuous and $r(t)>0$ and $a(t, s) \geq 0$ for $t>s$ and there exist rd-continuous functions $k, m: T \rightarrow R^{+}$such that

$$
\begin{gathered}
a(t, s) \leq k(t) m(s) \quad \forall t \geq s, \\
\sup _{t \geq 0} k(t)=: k_{1}<\infty, \\
\sup _{t \geq 0} \int_{0}^{t} m(s) \Delta s=k_{2}<\infty .
\end{gathered}
$$

(II) $F: T \times R \rightarrow R$ is continuous and assume that there exists rd-continuous function $q: T \rightarrow(0, \infty)$ and a real number $\beta$ with $0<\beta \leq 1$ such that

$$
x F(t, x) \leq q(t)|x|^{\beta+1} \quad \text { for } x \neq 0, t \geq 0 .
$$

We only consider those solutions of (1) which are nontrivial and delta-differentiable on $[0, \infty)$. The term solution henceforth applies to such solutions of (1).

A solution $x(t)$ of (1) is said to be oscillatory if, for every $t_{0}>0$, we have

$$
\inf _{t \geq t_{0}} x(t)<0<\sup _{t \geq t_{0}} x(t)
$$

and it is said to be nonoscillatory otherwise.

To the best of our knowledge, there are no results on the oscillatory behavior of solutions of (1). Therefore, the main goal of this paper is to establish some new criteria for the oscillatory behavior of (1) and other related equations. Also we provide some numerical examples to illustrate the results when $T=R$.

\section{Main Results}

We shall employ the following lemmas. 
Lemma 1 (see [6]). If $X$ and Yare nonnegative, then

$$
\begin{array}{ll}
X^{\lambda}+(\lambda-1) Y^{\lambda}-\lambda X Y^{\lambda-1} \geq 0 ; & \lambda>1, \\
X^{\lambda}-(1-\lambda) Y^{\lambda}-\lambda X Y^{\lambda-1} \leq 0 ; & \lambda<1,
\end{array}
$$

where equality holds if and only if $X=Y$.

This section is devoted to the study of the oscillatory properties of (1). In what follows, we let

$$
\begin{aligned}
g_{ \pm}(t)= & e(t) \mp k_{1}(1-\beta) \beta^{\beta /(1-\beta)} \\
& \times \int_{0}^{t} p^{\beta /(\beta-1)}(s) q^{1 /(1-\beta)}(s) m^{1 /(1-\beta)}(s) \Delta s ; \\
& 0<\beta<1 .
\end{aligned}
$$

Now we give sufficient conditions under which a nonoscillatory solution $x(t)$ of (1) satisfies

$$
x(t)=O(t) \quad \text { as } t \longrightarrow \infty .
$$

Theorem 2. Let $0<\beta<1$, conditions (I) and (II) hold, and for all $t_{0}>0$, the function $t / r(t)$ is bounded:

$$
\begin{aligned}
& \int_{t_{0}}^{\infty} \frac{s}{r(s)} \Delta s<\infty, \\
& \int_{t_{0}}^{\infty} s p(s) \Delta s<\infty .
\end{aligned}
$$

If

$$
\begin{gathered}
\limsup _{t \rightarrow \infty} \frac{1}{t} \int_{t_{0}}^{t} \frac{1}{r(u)} \int_{t_{0}}^{u} g_{-}(s) \Delta s \Delta u<\infty, \\
\liminf _{t \rightarrow \infty} \frac{1}{t} \int_{t_{0}}^{t} \frac{1}{r(u)} \int_{t_{0}}^{u} g_{+}(s) \Delta s \Delta u>-\infty,
\end{gathered}
$$

then every nonoscillatory solution $x(t)$ of (1) satisfies

$$
\limsup _{t \rightarrow \infty} \frac{|x(t)|}{t}<\infty
$$

Proof. Let $x(t)$ be a nonoscillatory solution of (1). First, assume $x(t)$ is eventually positive, say $x(t)>0$ for $t \geq t_{1}$ for some $t_{1} \geq 0$. Using condition (4) in (1) we have

$$
\begin{aligned}
\left(r(t) x^{\Delta}(t)\right)^{\Delta} \leq & e(t)-\int_{0}^{t_{1}} a(t, s) F(s, x(s)) \Delta s \\
& +\int_{t_{1}}^{t} a(t, s) q(s) x^{\beta}(s) \Delta s \text { for } t \geq t_{1} .
\end{aligned}
$$

Let

$$
c:=\max _{0 \leq t \leq t_{1}}|F(t, x(t))|<\infty .
$$

By assumption (3) we obtain

$$
\begin{aligned}
\left|-\int_{0}^{t_{1}} a(t, s) F(s, x(s)) \Delta s\right| & \leq \int_{0}^{t_{1}} a(t, s)|F(t, x(t))| \Delta s \\
& \leq c \int_{0}^{t_{1}} a(t, s) \Delta s \leq c k_{1} k_{2} \\
& =: b \text { for } t \geq t_{1} .
\end{aligned}
$$

Hence, from (13) we have

$$
\begin{aligned}
\left(r(t) x^{\Delta}(t)\right)^{\Delta} \leq & e(t)+b+k_{1} \\
& \times \int_{t_{1}}^{t}\left[m(s) q(s) x^{\beta}(s)-p(s) x(s)\right] \Delta s \\
& +k_{1} \int_{t_{1}}^{t} p(s) x(s) \Delta s \quad \text { for } t \geq t_{1} .
\end{aligned}
$$

Applying Lemma 1 to $m(s) q(s) x^{\beta}(s)-p(s) x(s)$ with

$$
\begin{aligned}
& \lambda=\beta, \quad X=(q m)^{1 / \beta} x, \\
& Y=\left(\frac{1}{\beta} p(q m)^{-1 / \beta}\right)^{1 /(\beta-1)},
\end{aligned}
$$

we have

$$
\begin{aligned}
m(s) q & (s) x^{\beta}(s)-p(s) x(s) \\
\leq & (1-\beta) \beta^{\beta /(\beta-1)} \\
& \quad \times p^{\beta(\beta-1)}(s) m^{1 /(1-\beta)}(s) q^{1 /(1-\beta)}(s) .
\end{aligned}
$$

Thus, we get

$$
\left(r(t) x^{\Delta}(t)\right)^{\Delta} \leq g_{+}(t)+b+k_{1} \int_{t_{1}}^{t} p(s) x(s) \Delta s \quad \text { for } t \geq t_{1} .
$$

Integrating (19) from $t_{1}$ to $t$ we have

$$
\begin{aligned}
r(t) x^{\Delta}(t) \leq & r\left(t_{1}\right) x^{\Delta}\left(t_{1}\right)+\int_{t_{1}}^{t} g_{+}(s) \Delta s+b\left(t-t_{1}\right) \\
& +k_{1} \int_{t_{1}}^{t} \int_{t_{1}}^{u} p(s) x(s) \Delta s \Delta u \text { for } t \geq t_{1} .
\end{aligned}
$$

Employing Lemma 3 in [7] to interchange the order of integration, we obtain

$$
\begin{aligned}
r(t) x^{\Delta}(t) \leq & r\left(t_{1}\right) x^{\Delta}\left(t_{1}\right)+\int_{t_{1}}^{t} g_{+}(s) \Delta s+b\left(t-t_{1}\right) \\
& +k_{1} t \int_{t_{1}}^{t} p(s) x(s) \Delta s \quad \text { for } t \geq t_{1},
\end{aligned}
$$

and so

$$
\begin{aligned}
x^{\Delta}(t) \leq & \frac{r\left(t_{1}\right) x^{\Delta}\left(t_{1}\right)}{r(t)}+\frac{1}{r(t)} \int_{t_{1}}^{t} g_{+}(s) \Delta s \\
& +\frac{b\left(t-t_{1}\right)}{r(t)}+\frac{k_{1} t}{r(t)} \int_{t_{1}}^{t} p(s) x(s) \Delta s \quad \text { for } t \geq t_{1} .
\end{aligned}
$$


Integrating this inequality from $t_{1}$ to $t$ and using (9) and the fact that the function $t / r(t)$ is bounded for $t \geq t_{1}$, say by $k_{3}$, we see that

$$
\begin{aligned}
x(t) \leq & x\left(t_{1}\right)+r\left(t_{1}\right) x^{\Delta}\left(t_{1}\right) \int_{t_{1}}^{t} \frac{1}{r(s)} \Delta s \\
& +\int_{t_{1}}^{t} \frac{1}{r(u)} \int_{t_{1}}^{u} g_{+}(s) \Delta s \Delta u+b \int_{t_{1}}^{t} \frac{s}{r(s)} \Delta s+k_{1} k_{3} \\
& \times \int_{t_{1}}^{t} \int_{t_{1}}^{u} p(s) x(s) \Delta s \Delta u \text { for } t \geq t_{1} .
\end{aligned}
$$

Once again, using Lemma 3 in [7] we have

$$
\begin{aligned}
x(t) \leq & x\left(t_{1}\right)+r\left(t_{1}\right) x^{\Delta}\left(t_{1}\right) \int_{t_{1}}^{t} \frac{1}{r(s)} \Delta s \\
& +\int_{t_{1}}^{t} \frac{1}{r(u)} \int_{t_{1}}^{u} g_{+}(s) \Delta s \Delta u+b \int_{t_{1}}^{t} \frac{s}{r(s)} \Delta s+k_{1} k_{3} t \\
& \times \int_{t_{1}}^{t} p(s) x(s) \Delta s \text { for } t \geq t_{1},
\end{aligned}
$$

or

$$
\begin{aligned}
\frac{x(t)}{t} \leq \frac{1}{t}\left[x\left(t_{1}\right)+r\left(t_{1}\right) x^{\Delta}\left(t_{1}\right) \int_{t_{1}}^{t} \frac{1}{r(s)} \Delta s\right. & \\
& \left.\quad+\int_{t_{1}}^{t} \frac{1}{r(u)} \int_{t_{1}}^{u} g_{+}(s) \Delta s \Delta u+b \int_{t_{1}}^{t} \frac{s}{r(s)} \Delta s\right] \\
& +k_{1} k_{3} \int_{t_{1}}^{t} p(s) x(s) \Delta s \text { for } t \geq t_{1},
\end{aligned}
$$

and so

$$
\frac{x(t)}{t} \leq c_{1}+c_{2} \int_{t_{1}}^{t} s p(s)\left(\frac{x(s)}{s}\right) \Delta s \text { for } t \geq t_{1},
$$

where $c_{2}=k_{1} k_{3}$ and $c_{1}$ is an upper bound for the expression

$$
\begin{aligned}
& \frac{1}{t}\left[x\left(t_{1}\right)+r\left(t_{1}\right) x^{\Delta}\left(t_{1}\right) \int_{t_{1}}^{t} \frac{1}{r(s)} \Delta s\right. \\
& \left.\quad+\int_{t_{1}}^{t} \frac{1}{r(u)} \int_{t_{1}}^{u} g_{+}(s) \Delta s \Delta u+b \int_{t_{1}}^{t} \frac{s}{r(s)} \Delta s\right] \quad \text { for } t \geq t_{1} .
\end{aligned}
$$

Applying Gronwall's inequality [1, Corollary 6.7] to inequality (26) and then using condition (10) we have

$$
\limsup _{t \rightarrow \infty} \frac{x(t)}{t}<\infty
$$

If $x(t)$ is eventually negative, we can set $y(t)=-x(t)$ to see that $y(t)$ satisfies (1) with $e(t)$ replaced by $-e(t)$ and $F(t, x)$ replaced by $-F(t,-y)$. It follows in a similar manner that

$$
\limsup _{t \rightarrow \infty} \frac{-x(t)}{t}<\infty .
$$

We conclude from (28) and (29) that (12) holds.
Next, by employing Theorem 2 we present the following oscillation result for (1).

Theorem 3. Let $0<\beta<1$, conditions (I), (II), (9), and (11) hold, and

$$
\operatorname{limsupt}_{t \rightarrow \infty} \int_{t_{0}}^{t} s p(s) \Delta s<\infty
$$

If

$$
\begin{aligned}
& \limsup _{t \rightarrow \infty} \int_{t_{0}}^{t} \frac{1}{r(u)} \int_{t_{0}}^{u} g_{-}(s) \Delta s \Delta u=\infty, \\
& \liminf _{t \rightarrow \infty} \int_{t_{0}}^{t} \frac{1}{r(u)} \int_{t_{0}}^{u} g_{+}(s) \Delta s \Delta u=-\infty,
\end{aligned}
$$

for all $t_{0} \geq 0$, then (1) is oscillatory.

Proof. Let $x(t)$ be a nonoscillatory solution of (1), say $x(t)>0$ for $t \geq t_{1}$ for some $t_{1} \geq 0$. The proof when $x(t)$ is eventually negative is similar. Proceeding as in the proof of Theorem 2 we arrive at

$$
\begin{aligned}
x(t) \leq & x\left(t_{1}\right)+r\left(t_{1}\right) x^{\Delta}\left(t_{1}\right) \int_{t_{1}}^{t} \frac{1}{r(s)} \Delta s \\
& +\int_{t_{1}}^{t} \frac{1}{r(u)} \int_{t_{1}}^{u} g_{+}(s) \Delta s \Delta u+b \int_{t_{1}}^{t} \frac{s}{r(s)} \Delta s+k_{1} k_{3} t \\
& \times \int_{t_{1}}^{t} s p(s)\left(\frac{x(s)}{s}\right) \Delta s \text { for } t \geq t_{1} .
\end{aligned}
$$

Condition (30) implies condition (10) and so the conclusion of Theorem 2 holds. This, together with (9), shows that the second and the last two integrals in (32) are bounded. Finally, taking limsup $\sup _{t \rightarrow \infty}$ and using conditions (30) and (31) result in a contradiction with the fact that $x(t)$ is eventually positive.

Corollary 4. Let $0<\beta<1$ and condition (I), (II), (9), and (30) hold. In addition, assume that

$$
\begin{gathered}
\limsup _{t \rightarrow \infty} \frac{1}{t} \int_{t_{0}}^{t} \frac{1}{r(u)} \int_{t_{0}}^{u} e(s) \Delta s \Delta u<\infty, \\
\liminf _{t \rightarrow \infty} \frac{1}{t} \int_{t_{0}}^{t} \frac{1}{r(u)} \int_{t_{0}}^{u} e(s) \Delta s \Delta u>-\infty,
\end{gathered}
$$

$$
\begin{aligned}
\lim _{t \rightarrow \infty} \frac{1}{t} \int_{t_{0}}^{t} \frac{1}{r(u)} \\
\quad \times \int_{t_{0}}^{u} p^{\beta /(\beta-1)}(s) q(s)^{1 /(1-\beta)} m^{1 /(1-\beta)}(s) \Delta s \Delta u<\infty .
\end{aligned}
$$

If

$$
\begin{gathered}
\limsup _{t \rightarrow \infty} \frac{1}{t} \int_{t_{0}}^{t} \frac{1}{r(u)} \int_{t_{0}}^{u} e(s) \Delta s \Delta u=\infty, \\
\liminf _{t \rightarrow \infty} \frac{1}{t} \int_{t_{0}}^{t} \frac{1}{r(u)} \int_{t_{0}}^{u} e(s) \Delta s \Delta u=-\infty,
\end{gathered}
$$


for all $t_{0} \geq 0$, then (1) is oscillatory. Similar reasoning to that in the sublinear case guarantees the following theorems for the integrodynamic (1) when $\beta=1$.

Theorem 5. Let $\beta=1$, and conditions (I) and (II) hold. In addition, suppose (9) and (33) and for all $t_{0} \geq 0$ assume that

$$
\limsup _{t \rightarrow \infty} \int_{t_{0}}^{t} \operatorname{sm}(s) q(s) \Delta s<\infty .
$$

Then every nonoscillatory solution of (1) satisfies (12).

Theorem 6. Let $\beta=1$, and conditions (I) and (II) hold. In addition, suppose (9) and (33) hold and assume that (35) is satisfied and for all $t_{0} \geq 0$,

$$
\limsup _{t \rightarrow \infty} t \int_{t_{0}}^{t} \operatorname{sm}(s) q(s) \Delta s<\infty .
$$

Then (1) is oscillatory.

Remark 7. The technique in this paper can be employed to Volterra integral equation on time scales of the form

$$
x(t)+\int_{0}^{t} a(t, s) F(s, x(s)) \Delta s=e(t) .
$$

Remark 8. We note that the results in this section can be obtained by using the additional assumption that the function $a(t, s)$ is nonincreasing with respect to the first variable. In this case the function $k_{1} m(t)$ which appeared in the proofs and the function $m(t)$ which appeared in the statements of the theorems are replaced by $a(t, t)$. The details are left to the reader.

\section{Illustrative Examples}

As a numerical illustration of our results of Section 2, we consider the following equation:

$$
\begin{array}{r}
\left((1+t)^{3} x^{\prime}(t)\right)^{\prime}+\int_{0}^{t} \frac{x^{\alpha}(s)}{\left(t^{2}+1\right)\left(s^{2}+1\right)} d s=e(t), \\
0<t \leq T_{f}
\end{array}
$$

with initial conditions $x(0)=x_{0}$ and $x^{\prime}(0)=x_{0}^{\prime}$. Equation (39) can be converted into two first order ordinary differential equations by substituting $(1+t)^{3} x^{\prime}(t)=y(t)$. This will lead to the following system:

$$
\begin{aligned}
& x^{\prime}(t)=\frac{y(t)}{(1+t)^{3}} ; \quad x(0)=x_{0}, \\
& y^{\prime}(t)=e(t)-\int_{0}^{t} \frac{x^{\alpha}(s)}{\left(t^{2}+1\right)\left(s^{2}+1\right)} d s ; \quad y(0)=x_{0}^{\prime} .
\end{aligned}
$$

Many numerical techniques can be used to solve (40). In the current work, the second-order accurate modified Euler technique is considered. The time interval $\left[0, T_{f}\right]$ will be subdivided into $N$ equal subdivisions with $\Delta t$ width for

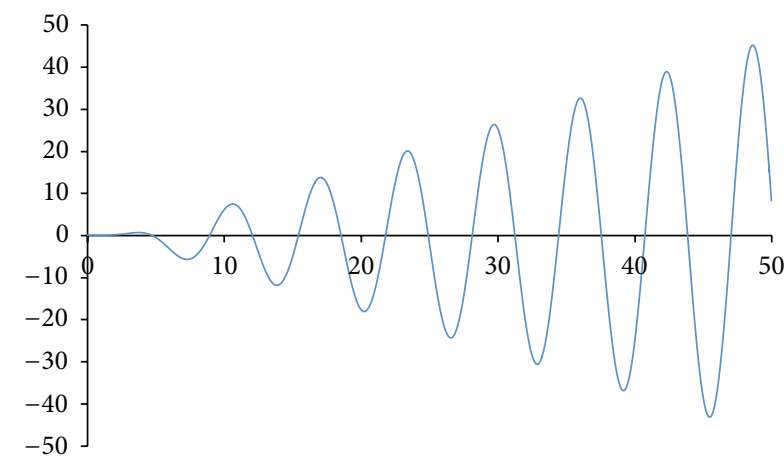

FIGURE 1: The numerical solution $x(t)$ for $e(t)=t^{4} \sin (t), \alpha=1 / 3$ and initial conditions $x(0)=0.1, x^{\prime}(0)=0$.

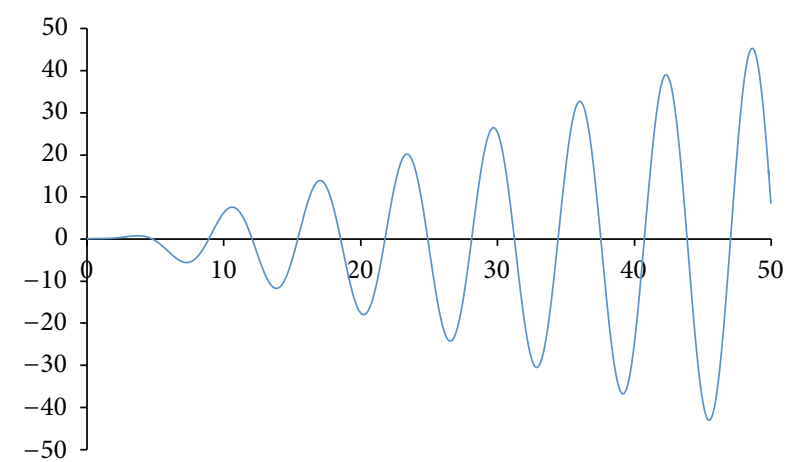

Figure 2: The numerical solution $x(t)$ for $e(t)=t^{4} \sin (t), \alpha=1$ and initial conditions $x(0)=0.1, x^{\prime}(0)=0.1$.

each subdivision. The prediction and correction steps of the modified Euler technique will be

$$
\begin{aligned}
& \bar{x}_{i+1}=x_{i}+\Delta \operatorname{tg}\left(t_{i}, x_{i}, y_{i}\right), \\
& \bar{y}_{i+1}=y_{i}+\Delta \operatorname{th}\left(t_{i}, x_{i}, y_{i}\right), \\
& x_{i+1}=x_{i}+0.5 \Delta t\left[g\left(t_{i}, x_{i}, y_{i}\right)+g\left(t_{i+1}, \bar{x}_{i+1}, \bar{y}_{i+1}\right)\right], \\
& y_{i+1}=y_{i}+0.5 \Delta t\left[h\left(t_{i}, x_{i}, y_{i}\right)+h\left(t_{i+1}, \bar{x}_{i+1}, \bar{y}_{i+1}\right)\right],
\end{aligned}
$$

where

$$
\begin{aligned}
& g(t, x, y)=\frac{y(t)}{(1+t)^{3}} \\
& h(t, x, y)=e(t)-\int_{0}^{t} \frac{x^{\alpha}(s)}{\left(t^{2}+1\right)\left(s^{2}+1\right)} d s .
\end{aligned}
$$

The integral (43) can be approximated numerically at each time instant $t_{i}$ using the trapezoidal rule, which has accuracy of $O(\Delta t)^{2}$.

Let $T_{f}=50, x(0)=0.1$ and $x^{\prime}(0)=0$, with $\alpha=1 / 3$. For $e(t)=t^{4} \sin (t)$, all conditions of Theorems 3 and 6 are satisfied and hence (39) is oscillatory (see Figure 1). In Figure 2, the initial conditions are changed to be $x(0)=0.1$ and $x^{\prime}(0)=0.1$ with $\alpha=1$; the solution is also oscillatory. 


\section{Conclusions}

The oscillatory behavior of a second-order integrodynamic equation on time scales is considered. The results in the paper establish new criteria and it is shown that these results can be employed for Volterra integral equation on time scale. A numerical example in the continuous case is solved to show the validity of the results.

\section{Conflict of Interests}

The authors declare that there is no conflict of interests regarding the publication of this paper.

\section{References}

[1] M. Bohner and A. Peterson, Dynamic Equations on Time-Scales: An Introduction with Applications, Birkhäauser, Boston, Mass, USA, 2001.

[2] G. Karakostas, I. P. Stavroulakis, and Y. Wu, "Oscillations of Volterra integral equations with delay," The Tohoku Mathematical Journal, vol. 45, no. 4, pp. 583-605, 1993.

[3] H. Onose, "On oscillation of Volterra integral equations and first order functional-differential equations," Hiroshima Mathematical Journal, vol. 20, no. 2, pp. 223-229, 1990.

[4] N. Parhi and N. Misra, "On oscillatory and nonoscillatory behaviour of solutions of Volterra integral equations," Journal of Mathematical Analysis and Applications, vol. 94, no. 1, pp. 137149, 1983.

[5] B. Singh, "On the oscillation of a Volterra integral equation," Czechoslovak Mathematical Journal, vol. 45, no. 4, pp. 699-707, 1995.

[6] G. H. Hardy, I. E. Littlewood, and G. Pólya, Inequalities, Cambridge University Press, Cambridge, Mass, USA, 1959.

[7] M. Bohner and S. Stević, "Asymptotic behavior of second-order dynamic equations," Applied Mathematics and Computation, vol. 188, no. 2, pp. 1503-1512, 2007. 


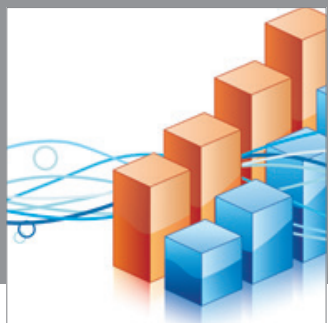

Advances in

Operations Research

mansans

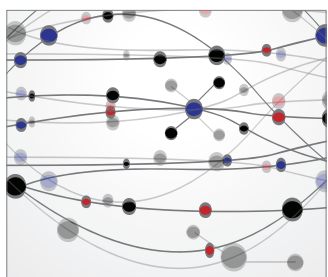

The Scientific World Journal
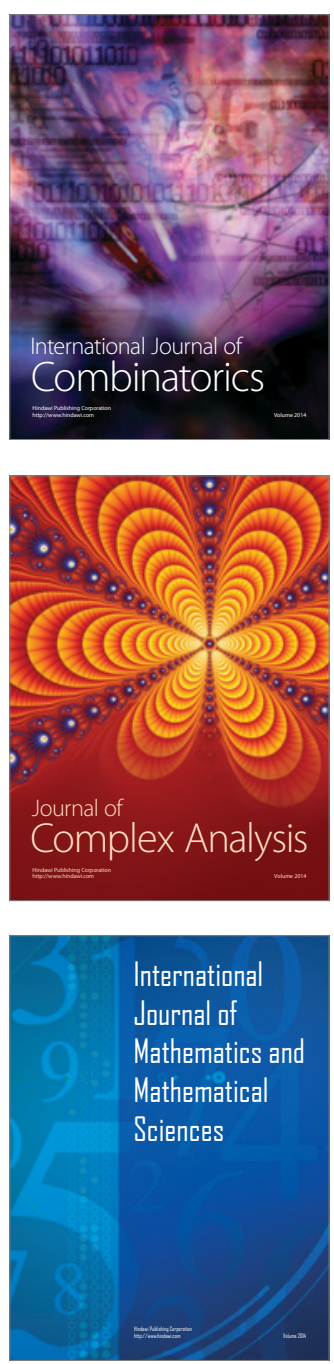
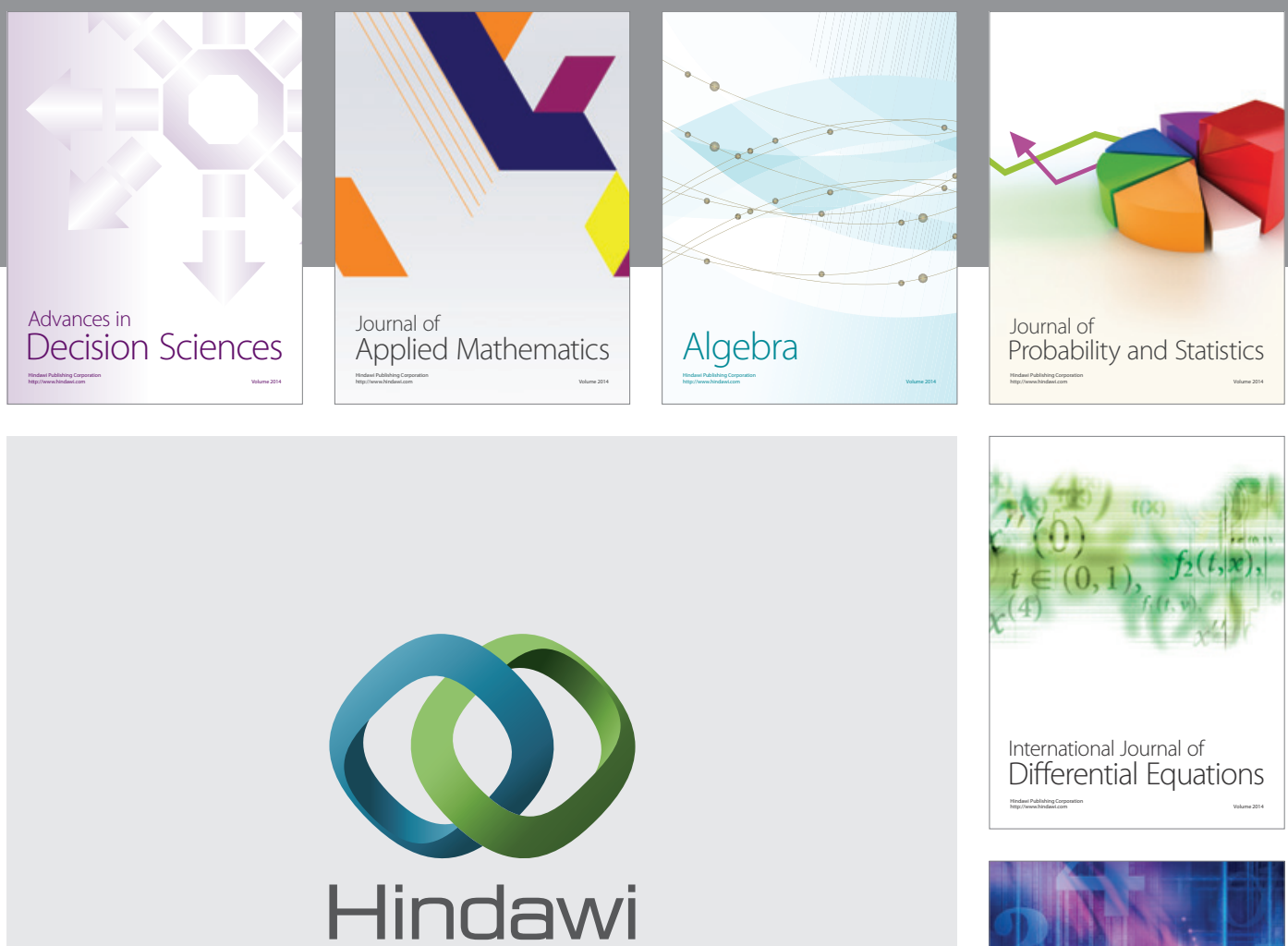

Submit your manuscripts at http://www.hindawi.com
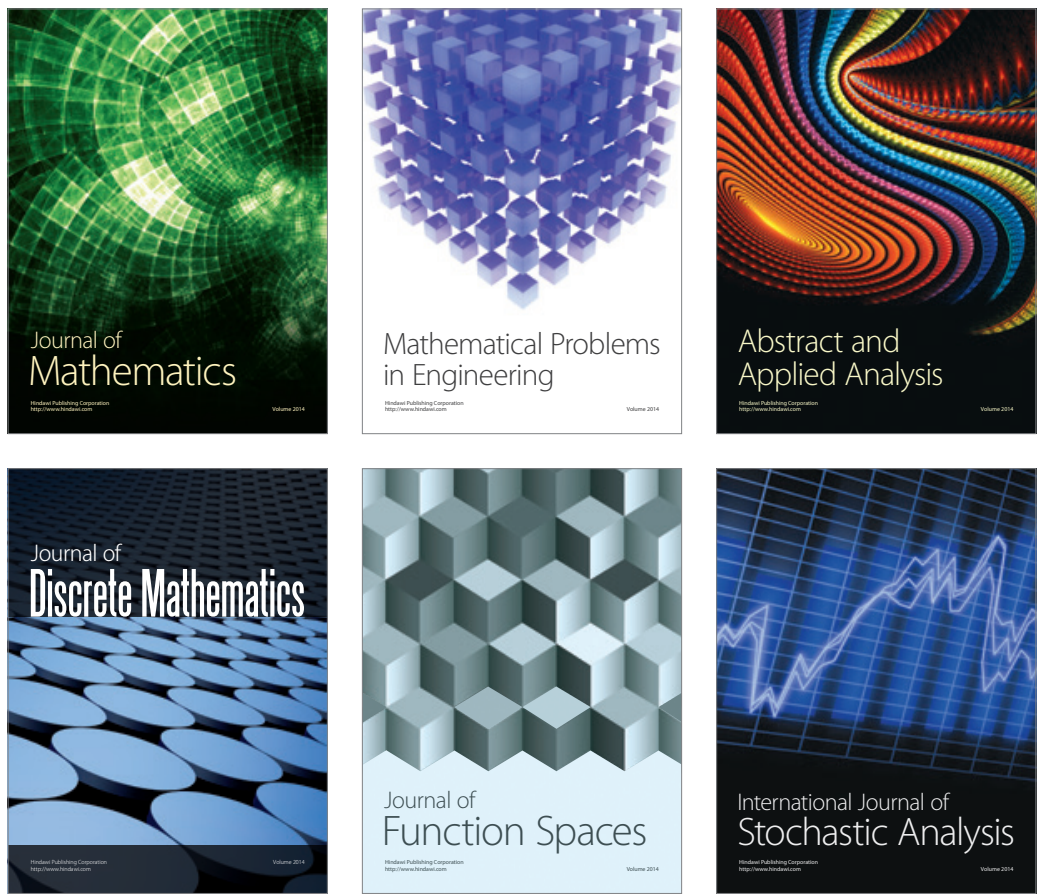

Journal of

Function Spaces

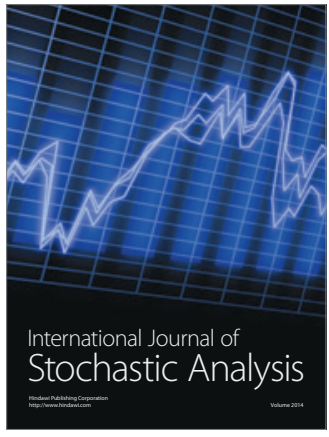

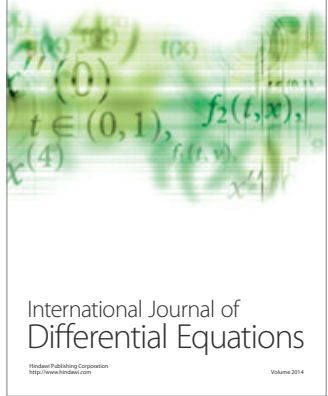
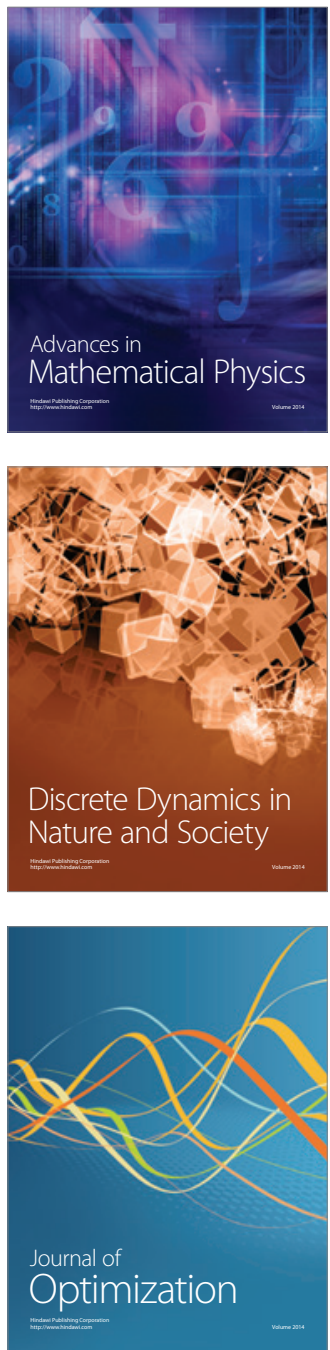